\title{
SEASONAL ACTIVITY OF FIORINIA DATE SCALE, FIORINIA PHOENICIS BALACHOWSKY (HEMIPTERA - DIASPIDIDAE) ON DATE PALM AT GIZA GOVERNORATE, EGYPT
}

\author{
ELWAN, E. A., MAHA I. EL-SAYED AND A. M. SERAG
}

Plant Protection Research Institute, ARC, Dokki, Giza

Email: Dr.Sayedelwan@Yahoo.com

(Manuscript received 5 January 2011)

\begin{abstract}
The seasonal activity of fiorinia date scale, Fiorinia phoenicis Balachowsky, 1967 (Hemiptera - Diaspididae) was studied for two years (March, 2008 until mid-February, 2010) on date palm at Giza governorate. The obtained results revealed that $F$. phoenicis has three overlapping generations under field conditions. The $1^{\text {st }}$ generation (early summer generation) peaked in early June and the $2^{\text {nd }}$ generation (late summer generation) peaked around August/September whereas the $3^{\text {rd }}$ generation (autumn generation) peaked in October/November. The shortest generation was the late summer generation (2.5 - 3.0 months) at $27.9-28.6^{\circ} \mathrm{C}$ and $63.5-$ $64.8 \%$ R.H.whereas the longest one was autumn generation (4.5 months) at $21.6-22.0^{\circ} \mathrm{C}$ and $65.9-66.3 \%$ R.H. The early summer generation had intermediate duration (4.0 - 4.5 months) at 20.9 $21.2^{\circ} \mathrm{C}$ and $62.5-63.0 \%$ R.H in the two years, respectively. The population density was varied in the three generations, the autumn generation was the largest one with total population ranged 944.7 1016.0 insects/leaflet with mean of 980.4 insects/leaflet followed by late summer generation 829.0 - 1025.7 insects/leaflet with mean of 927.4 insects/leaflet whereas the smallest one was the early summer generation (422.4 - 575.7 insects/leaflet with mean of 499.1 insects/leaflet). The insect population occurred on date palm fronds all the year round and decreased to lower numbers during winter season and this referred to the cold weather. The basal stratum of date palm leaflets received the highest infestation $(37.4-38.3 \%)$ than middle (34.4 - 35.3\%) and apical ones (26.4 - 28.2\%).

On the other hand, the insect activity affected significantly with daily mean temperature and \%R.H. in the both years. The combined effect of daily mean temperature and \%R.H on nymph and adult populations showed $66.1-69 \% \& 48.1-49.2 \%$ in the $1^{\text {st }}$ generation, $65.4-74.0 \% \& 63.8-78.4 \%$ in the $2^{\text {nd }}$ generation and $60.9-$ $77.4 \% \& 48.6-63.5 \%$ in the $3^{\text {rd }}$ generation in the both years, respectively.
\end{abstract}

\section{INTRODUCTION}

Date palm (Phoenix dactylifera L.) is economic important tree in many countries and many industries have been initiated on the dates as well as on many parts of date palm trees. The insect pests of date palm were studied by many researchers such as Martin, (1958), Hussain (1974), El-Haidari (1980), El-Haidari \& Al-Hafidh (1986), Elwan (2000) and Abdel-Rahman \& Amro (2007). 
In last few years, scale insect was observed with high numbers on date palm fronds and dates in some Governorates in Egypt causing yellowish of the pinnae and dryness of the fronds as well as the infested dates becomes unmarketable and has a lower grade. The scale insect was collected and identified in the Department of Scale insects and Mealy bugs, Plant Protection Research Institute as Fiorinia date scale, Fiorinia phoenicis Balachowsky, 1967 (Hemiptera: Diaspididae) a new pest on date palm in Egypt (Ghabbour and Mohammad, 2010). Fiorinia date scale, F. phoenicis was recorded in Iraq (Hussain, 1974), Saudi Arabia (Matile, 1984), Oman (Ghabbour et al., 1996) and Iran (Takagi \& Moghaddam, 2005).

F. phoenicis infests the date palm fronds, specially the older ones as well as the dates. When the insect population increases, the scales move to the older fronds and date bunches then the younger fronds. The severe infestation affected greatly on the growth of date palm trees specially the offshoots and the quality of the infested dates become less marketable.

The present investigation was directed to $F$. phoenicis to study the seasonal activity, number and duration of generations, distribution of populations on date palm leaflets as well as effect of main weather factors on the insect activity.

\section{MATERIALS AND METHODS}

The present study was conducted on date palm trees cultivated in Agricultural Research Center, Giza Governorate for two years starting from March, 2008 until midFebruary, 2010. The selected date palm trees received the normal agricultural practices without pruning the fronds and application any chemical control measures before and during the period of investigation.

Four date palm trees similar in age, vegetation and height were randomly selected and sampling was practiced at half monthly intervals. A random sample of 20 leaflets was taken from the cardinal directions of each date palm at a rate of 5 leaflets per each direction i.e. North, South, East and West, respectively. The collected Leaflets were divided into three equal stratum (basal, middle and apical) then preserved in paper bags and transferred to the laboratory for inspection with stereoscopic-microscope, the insect population was counted and sorted to nymphs and adults. The half-monthly means of nymphs and adults per/leaflet were graphically illustrated.

Number and duration of annual generations under field conditions were determined by integration of the population curves in these figures. Distribution of $F$. phoenicis population on the date palm leaflets was determined. The Meteorological data were obtained from Giza Meteorological Station and the half monthly means of 
temperature and \%R.H. were estimated. The statistical analyses of the present work were carried out with Computer (MSTATC Program).

\section{RESULTS AND DISCUSSION}

\section{1- Seasonal activity of $F$. phoenicis on date palm trees}

Data illustrated in Figs. (1\&2) showed the half-monthly variation in the seasonal activity of the nymphs and adults of F. phoenicis at Giza Governorate for two years (March, 2008 until Mid-February, 2010). The trends of fluctuation in nymph and adult populations were almost similar. Accordingly, it's better to discuss the seasonal activity on the basis of average number of nymphs and adults counts at the successive sampling dates.

\section{A- Seasonal activity of $F$. phoenicis in the $\mathbf{1}^{\text {st }}$ year}

The initial mean count of nymph and adult populations in March (Fig. 1) ranged 120-150 and 130 - 192 insects/leaflet for nymph and adult populations, respectively. This count gradually increased in April and May. In early June, the population of nymphs and adults increased recording the $1^{\text {st }}$ peak with mean population of 670 and 510 insects/leaflet for nymphs and adults under field conditions of $24.7^{\circ} \mathrm{C}$ and $61.3 \%$ R.H., respectively. In July, the population gradually decreased and increased again through August with relatively high population.
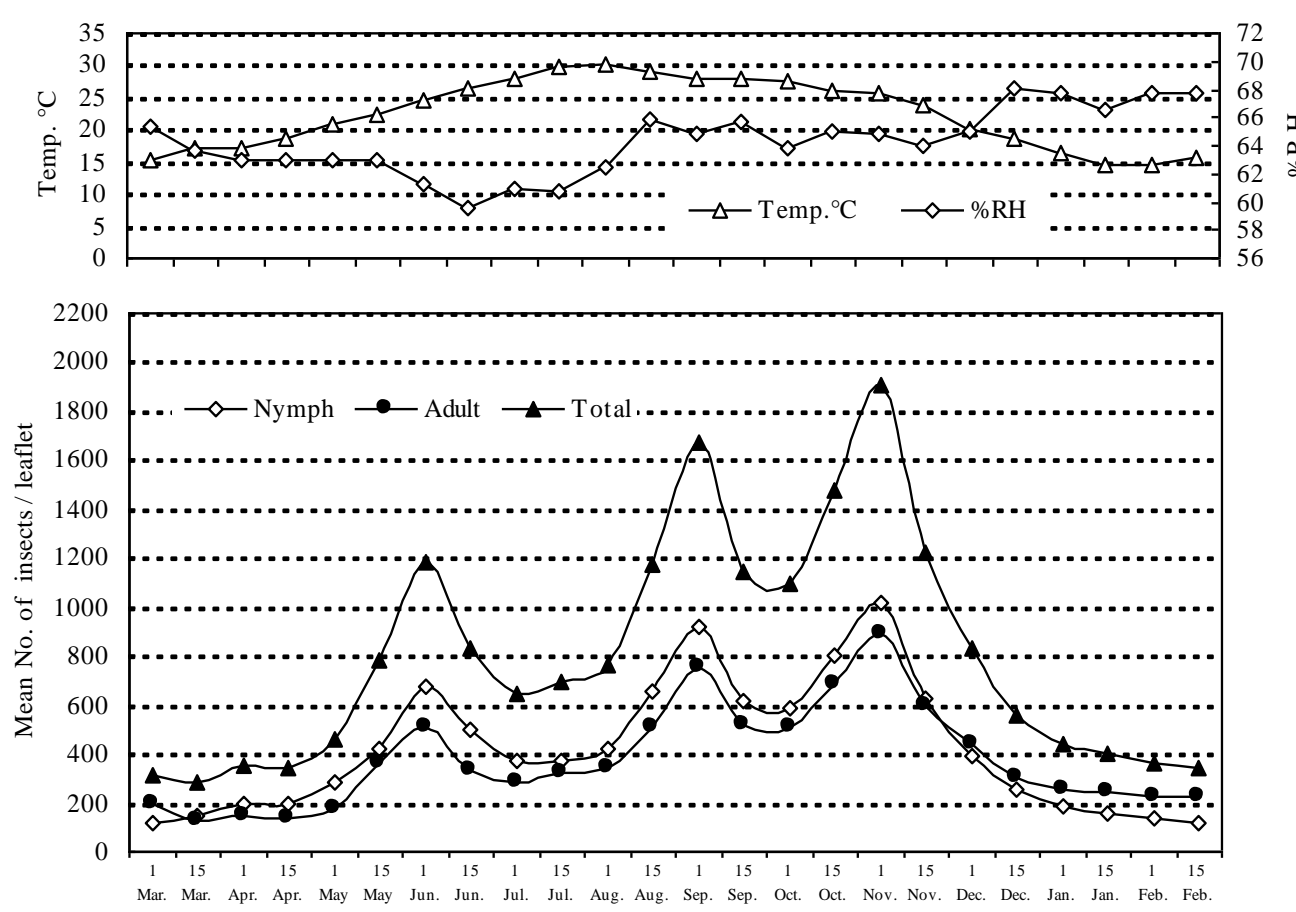

Fig. 1. Half-monthly means of $F$. phoenicis population/leaflet on date palm trees with corresponding half-monthly means of temperature and \%R.H. at Giza Governorate in the $1^{\text {st }}$ year $(2008 / 2009)$. 
In early September, the insect population increased and reached the $2^{\text {nd }}$ peak with mean population of 920 and 750 insects / leaflet for nymph and adult at $28.0^{\circ} \mathrm{C}$ and $64.8 \%$ R.H., respectively. The nymph and adult populations gradually decreased during mid-September and early October. In mid-October, the population of nymphs and adults gradually increased and reached to highest numbers by early November recording the $3^{\text {rd }}$ peak for insect activity with mean population of 1240 and 890 insects/leaflet at $25.8^{\circ} \mathrm{C}$ and $64.9 \%$ R.H., respectively. This peak was followed by a quick decline in insect population during December. Continues decrease occurred in insect population during January and February, the nymph and adult populations decreased to 160 - 115 and 254 - 225 insects/ leaflet, respectively.

\section{B- Seasonal activity of $F$. phoenicis in the $2^{\text {nd }}$ year}

The population of nymphs and adults was relatively low in March and April. In early May, the population gradually increased and reached to the $1^{\text {st }}$ peak by early June with mean population of 471 and 386 for nymphs and adults at $23.5^{\circ} \mathrm{C}$ and $61.9 \%$ R.H., respectively. Gradual decrease in the insect population was observed in July followed by gradual increase in early August. In mid-August, the insect population highly increased recording the $2^{\text {nd }}$ peak with mean population of 726 and 600 insects/leaflet for both nymph and adult populations at $28.6^{\circ} \mathrm{C}$ and $65.8 \%$ R.H., respectively.

In early September, the population gradually decreased and increased again by mid-September whereas in October the nymph and adult populations increased highly recording the $3^{\text {rd }}$ peak in mid-October with mean population of 1160 and 970 insects/leaflet at $25.8^{\circ} \mathrm{C}$ and $65.1 \%$ R.H. This peak was followed by gradual decline in nymph and adult populations during November and December. A continuous decrease was observed in insect population during January and February, ranged 195-150 and 250-210 insects/ leaflet, respectively. 

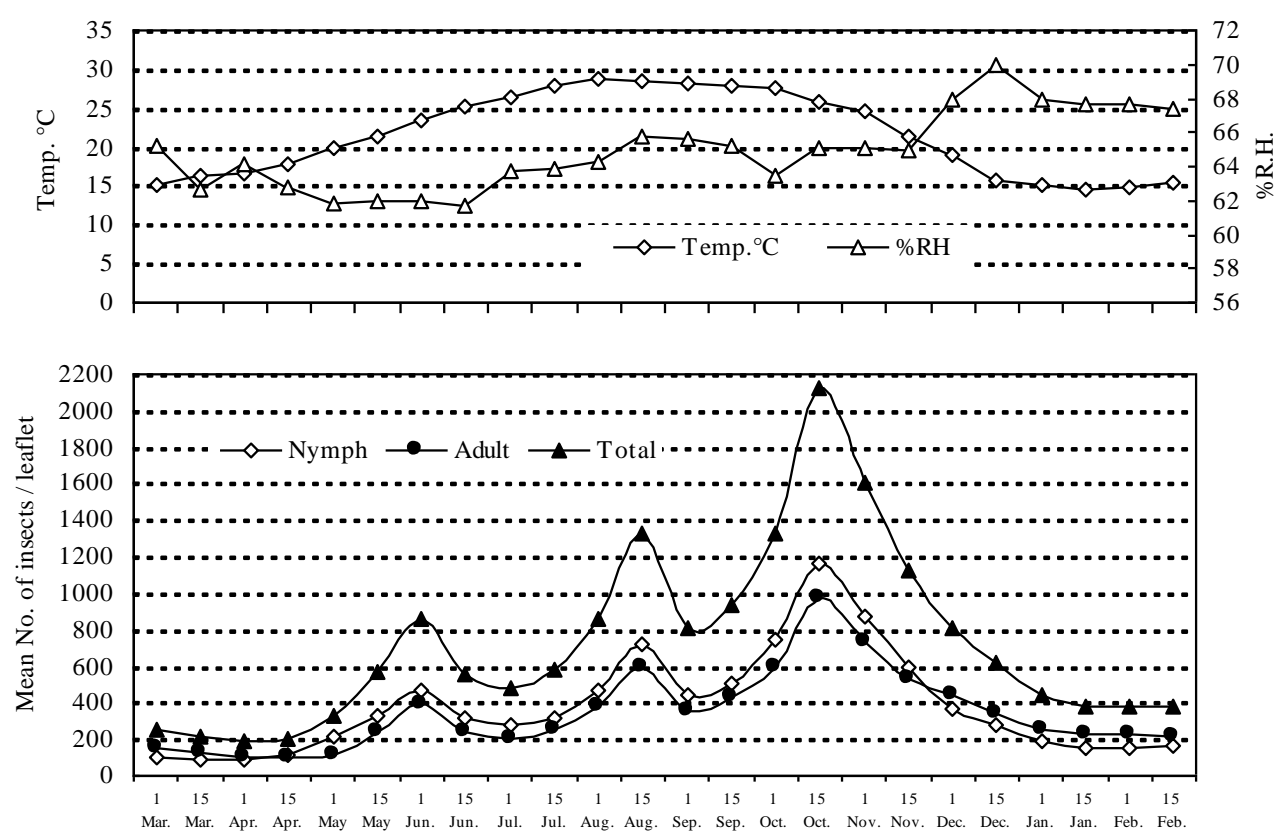

Fig. 2. Half-monthly means of F. phoenicis population/leaflet on date palm trees with corresponding half-monthly means of temperature and \%R.H. at Giza Governorate in the 2nd year (2009/2010).

The afore-mentioned results showed that fiorinia date scale, F. phoenicis has three overlapping generations under field conditions at Giza Governorate. The $1^{\text {st }}$ generation (early summer) peaked in early June and the $2^{\text {nd }}$ generation (late summer) peaked around August/September whereas the $3^{\text {rd }}$ generation (autumn generation) peaked in October/November. Also, the obtained results showed that, the insect population occurred on date palm fronds with lower numbers during winter season and this referred to the cold weather.

In this respect, parlatoria date scale Parlatoria blanchardii has similar activity on date palm. In Morocco, Smirnoff (1957) stated that, the development of $P$. blanchardii on date palm is continuous all the year round except for the period of winter diapause (January - February). The same results were observed in Pakistan (Sharif \& Wajih, 1982) and the infestation with $P$. blanchardii on date palm is vigorous through the year with some reduction in activity during winter months.

\section{1- Number and duration of annual generations of $F$. phoenicis under field conditions}

Data in Tables ( $1 \& 2)$ and Figs. ( $3 \& 4$ ) indicated that F. phoenicis has three overlapping generations a year. These generations occurred in early summer, late summer, and autumn as follows: 


\section{1- The $\mathbf{1}^{\text {st }}$ generation (early summer generation)}

The $1^{\text {st }}$ generation started from early March in the both years until early July in the $1^{\text {st }}$ year and mid-July in the $2^{\text {nd }}$ one. The generation peaked in early June in the two studied years and lasted for $4.0-4.5$ months at $20.9-21.2{ }^{\circ} \mathrm{C}$ and $62.5-$ $63.0 \%$ R.H, in the both years, respectively. The generation density ranged 323.3 230.8 nymphs/leaflet with average of 277.1 nymphs/leaflet and 191.6 - 252.4 adults/leaflet with average of 222.0 adults/leaflet, respectively.

\section{2- The $\mathbf{2}^{\text {nd }}$ generation (late summer generation)}

The $2^{\text {nd }}$ generation started in early July in the both years and continued until midSeptember in the $2^{\text {nd }}$ year and lasted for early October in the $1^{\text {st }}$ year. The generation peaked in mid-August in the both years. The $2^{\text {nd }}$ generation ranged $2.5-3.0$ months at $27.9-28.6^{\circ} \mathrm{C}$ and $63.5-64.8 \%$ R.H in the two years, respectively. The generation density ranged 457.2 - 564.3 nymphs/leaflet with average of 510.8 nymphs/leaflet and 3371.8 - 461.4 adults/leaflet with average of 416.6 adults/leaflet, respectively.

\section{3- The $3^{\text {rd }}$ generation (autumn generation)}

The $3^{\text {rd }}$ generation occurred between mid-September and early February in the $1^{\text {st }}$ year and from early September to mid-January in the $2^{\text {nd }}$ year. This generation peaked in mid-October or early November in the two years. The generation duration lasted for 4.5 months in the both years under field conditions ranged $21.6-22.0^{\circ} \mathrm{C}$ and 65.9 $66.3 \%$ R.H in the two years. The generation density ranged 478.4 - 528.8 nymphs/leaflet with average of 503.6 nymphs / leaflet and $466.3-487.2$ adults/leaflet with average of 476.8 adults / leaflet in the both years, respectively.

Table 1. Number and duration of annual generations of $F$. phoenicis on date palm trees at Giza, Governorate in the $1^{\text {st }}$ year $(2008 / 2009)$.

\begin{tabular}{|c|c|c|c|c|c|c|c|c|c|c|}
\hline \multirow{2}{*}{ Generation } & \multirow{2}{*}{$\begin{array}{l}\text { Insect } \\
\text { stage }\end{array}$} & \multicolumn{4}{|c|}{ Generation duration } & \multicolumn{3}{|c|}{ Generation density } & \multirow{2}{*}{$\begin{array}{c}\text { Mean } \\
{ }^{\circ} \mathrm{C}\end{array}$} & \multirow{2}{*}{$\begin{array}{c}\text { R.H } \\
\%\end{array}$} \\
\hline & & From & To & Peak & $\begin{array}{l}\text { Duration } \\
\text { / month }\end{array}$ & Nymph & Adult & Total & & \\
\hline \multirow{2}{*}{$\begin{array}{c}1^{\text {st }} \\
\text { Generation }\end{array}$} & Nymph & $\begin{array}{l}\text { Early } \\
\text { Mar. }\end{array}$ & $\begin{array}{l}\text { Early } \\
\text { Jul. }\end{array}$ & $\begin{array}{l}\text { Early } \\
\text { Jun. }\end{array}$ & 4 & \multirow{2}{*}{323.3} & \multirow{2}{*}{252.4} & \multirow{2}{*}{575.7} & \multirow{2}{*}{21.2} & \multirow{2}{*}{62.5} \\
\hline & Adult & $\begin{array}{l}\text { Early } \\
\text { Mar. }\end{array}$ & $\begin{array}{l}\text { Early } \\
\text { Jul. }\end{array}$ & $\begin{array}{l}\text { Early } \\
\text { Jun }\end{array}$ & 4 & & & & & \\
\hline \multirow{2}{*}{$\begin{array}{c}2^{\text {nd }} \\
\text { Generation }\end{array}$} & Nymph & $\begin{array}{l}\text { Early } \\
\text { Jul. }\end{array}$ & $\begin{array}{l}\text { Early } \\
\text { Oct. }\end{array}$ & $\begin{array}{l}\text { Mid- } \\
\text { Aug. }\end{array}$ & 3 & \multirow{2}{*}{564.3} & \multirow{2}{*}{461.4} & \multirow{2}{*}{1025.7} & \multirow{2}{*}{28.6} & \multirow{2}{*}{63.5} \\
\hline & Adult & $\begin{array}{l}\text { Early } \\
\text { Jul. }\end{array}$ & $\begin{array}{l}\text { Early } \\
\text { Oct. }\end{array}$ & $\begin{array}{l}\text { Mid- } \\
\text { Aug. }\end{array}$ & 3 & & & & & \\
\hline \multirow{2}{*}{$\begin{array}{c}3^{\text {rd }} \\
\text { Generation }\end{array}$} & Nymph & $\begin{array}{l}\text { Mid- } \\
\text { Sep. }\end{array}$ & $\begin{array}{l}\text { Early } \\
\text { Feb. }\end{array}$ & $\begin{array}{l}\text { Early } \\
\text { Nov. }\end{array}$ & 4.5 & \multirow{2}{*}{478.4} & \multirow{2}{*}{466.3} & \multirow{2}{*}{944.7} & \multirow{2}{*}{21.6} & \multirow{2}{*}{65.9} \\
\hline & Adult & $\begin{array}{l}\text { Mid- } \\
\text { Sep. }\end{array}$ & $\begin{array}{l}\text { Early } \\
\text { Feb. }\end{array}$ & $\begin{array}{l}\text { Early } \\
\text { Nov. }\end{array}$ & 4.5 & & & & & \\
\hline
\end{tabular}




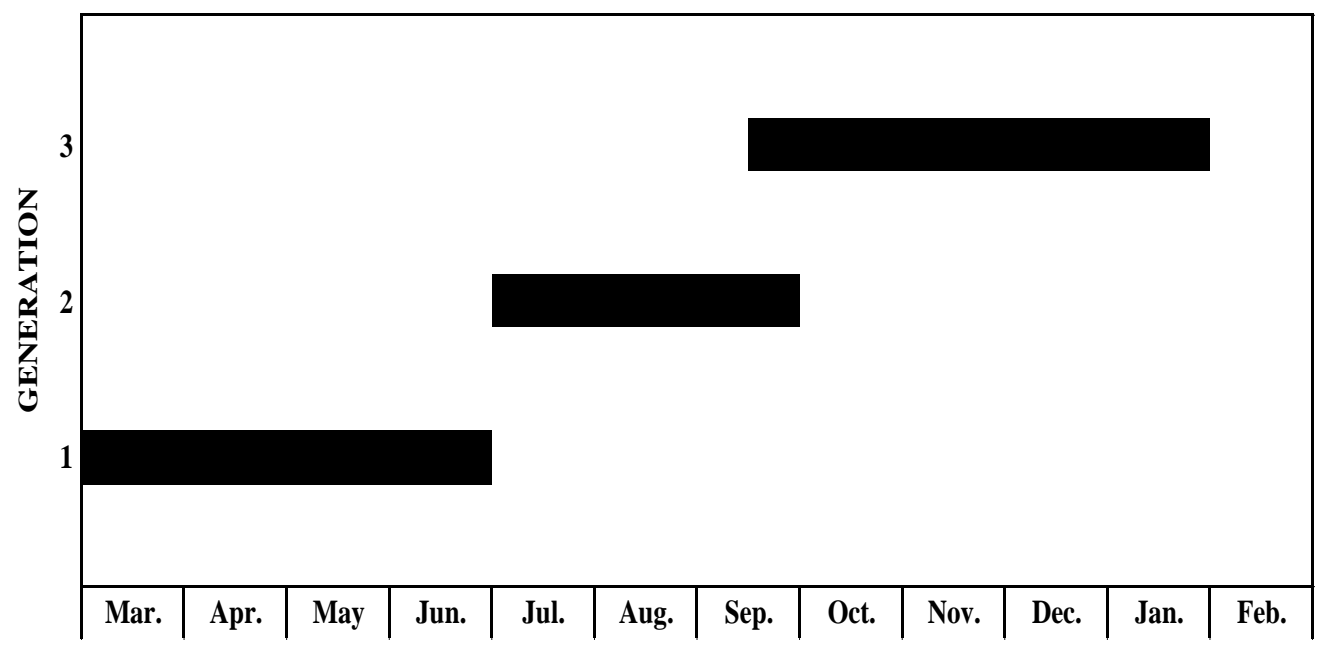

Fig. 3. Number and duration of annual generations of $F$. phoenicis on date palm trees at Giza, Governorate during the $1^{\text {st }}$ year (2008/2009).

Table 2. Number and duration of annual generations of $F$. phoenicis on date palm trees at Giza Governorate in the $2^{\text {nd }}$ year $(2009 / 2010)$.

\begin{tabular}{|c|c|c|c|c|c|c|c|c|c|c|}
\hline \multirow{2}{*}{ Generation } & \multirow{2}{*}{$\begin{array}{l}\text { Insect } \\
\text { stage }\end{array}$} & \multicolumn{4}{|c|}{ Generation period } & \multicolumn{3}{|c|}{ Generation density } & \multirow{2}{*}{$\begin{array}{c}\text { Mean } \\
{ }^{\circ} \mathrm{C} \\
\end{array}$} & \multirow{2}{*}{$\begin{array}{l}\text { R.H } \\
\%\end{array}$} \\
\hline & & From & To & Peak & $\begin{array}{l}\text { Duration } \\
\text { / month }\end{array}$ & Nymph & Adult & Total & & \\
\hline $1^{\text {st }}$ & Nymph & $\begin{array}{l}\text { Early } \\
\text { Mar. }\end{array}$ & $\begin{array}{l}\text { Mid- } \\
\text { Jul. }\end{array}$ & $\begin{array}{l}\text { Early } \\
\text { Jun. }\end{array}$ & 4.5 & \multirow{2}{*}{230.8} & \multirow{2}{*}{191.6} & \multirow{2}{*}{422.4} & \multirow{2}{*}{20.9} & \multirow{2}{*}{63.0} \\
\hline Generation & Adult & $\begin{array}{l}\text { Early } \\
\text { Mar. }\end{array}$ & $\begin{array}{l}\text { Mid- } \\
\text { Jul. }\end{array}$ & $\begin{array}{l}\text { Early } \\
\text { Jun. }\end{array}$ & 4.5 & & & & & \\
\hline \multirow{2}{*}{$\begin{array}{c}2^{\text {nd }} \\
\text { Generation }\end{array}$} & Nymph & $\begin{array}{c}\text { Early } \\
\text { Jul. }\end{array}$ & $\begin{array}{l}\text { Mid- } \\
\text { Sep. }\end{array}$ & $\begin{array}{l}\text { Mid- } \\
\text { Aug. }\end{array}$ & 2.5 & \multirow{2}{*}{457.2} & \multirow{2}{*}{371.8} & \multirow{2}{*}{829.0} & \multirow{2}{*}{27.9} & \multirow{2}{*}{64.8} \\
\hline & Adult & $\begin{array}{c}\text { Early } \\
\text { Jul. }\end{array}$ & $\begin{array}{l}\text { Mid- } \\
\text { Sep. }\end{array}$ & $\begin{array}{l}\text { Mid- } \\
\text { Aug. }\end{array}$ & 2.5 & & & & & \\
\hline \multirow{2}{*}{$\begin{array}{c}3^{\text {rd }} \\
\text { Generation }\end{array}$} & Nymph & $\begin{array}{l}\text { Early } \\
\text { Sep. }\end{array}$ & $\begin{array}{l}\text { Mid- } \\
\text { Jan. }\end{array}$ & $\begin{array}{l}\text { Mid- } \\
\text { Oct. }\end{array}$ & 4.5 & \multirow{2}{*}{528.8} & \multirow{2}{*}{487.2} & \multirow{2}{*}{1016.0} & \multirow{2}{*}{22.0} & \multirow{2}{*}{66.3} \\
\hline & Adult & $\begin{array}{l}\text { Early } \\
\text { Sep. }\end{array}$ & $\begin{array}{l}\text { Mid- } \\
\text { Jan. }\end{array}$ & $\begin{array}{l}\text { Mid- } \\
\text { Oct. }\end{array}$ & 4.5 & & & & & \\
\hline
\end{tabular}




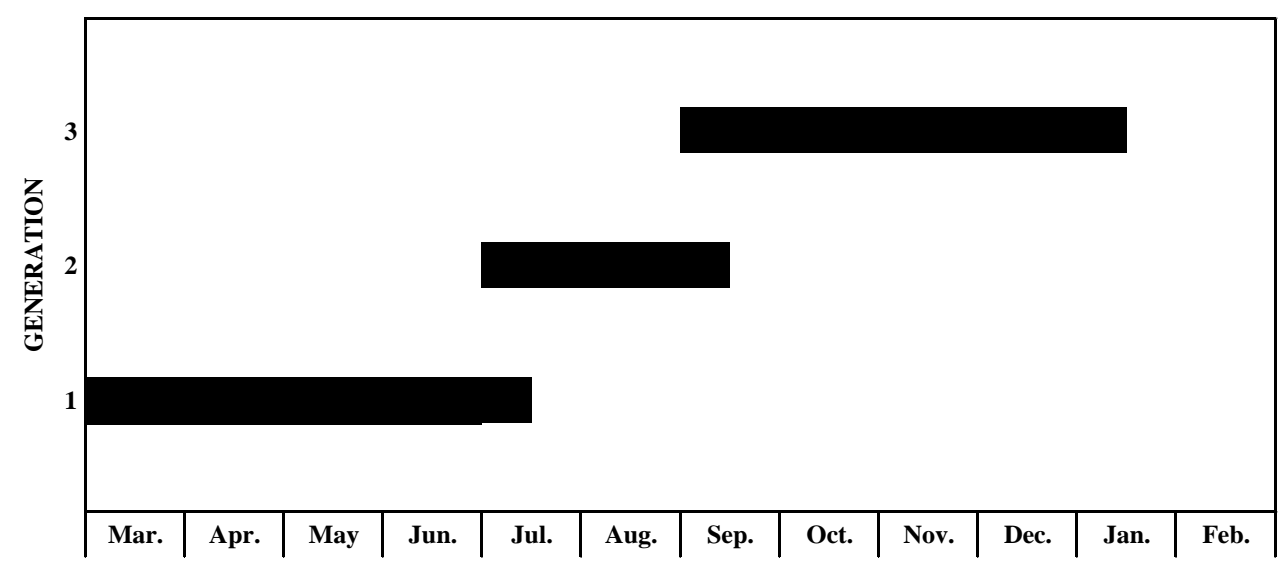

Fig. 4. Number and duration of annual generations of $F$. phoenicis on date palm trees at Giza Governorate in the $2^{\text {nd }}$ year (2009/2010).

The obtained results revealed that, the generation duration of $F$. phoenicis were varied in the both years. The shortest generation (late summer generation) occurred in summer ( $2.5-3.0$ months) at $27.9-28.6^{\circ} \mathrm{C}$ and $63.5-64.8 \%$ R.H. whereas the longest one (autumn generation) occurred in autumn (4.5 months) at $21.6-22.0^{\circ} \mathrm{C}$ and 65.9 - 66.3 \%R.H. The early summer generation has intermediate duration (4.0 4.5 months) at $20.9-21.2^{\circ} \mathrm{C}$ and $62.5-63.0 \% \mathrm{R} . \mathrm{H}$ in the two years, respectively.

On the other hand, the population density was varied in the generations, the autumn generation was the largest one with total population ranged $944.7-1016.0$ insects/leaflet with mean of 980.4 insects/leaflet followed by late summer generation (829.0 - 1025.7 insects/leaflet with mean of 927.4 insects/leaflet) whereas the smallest density occurred in the early summer generation (422.4 - 575.7 insects/leaflet with mean of 499.1 insects/leaflet), respectively.

The above mentioned results showed that, fiorinia date scale, F. phoenicis has three overlapping generations a year under environmental conditions of Giza Governorate. In this respect, Talhouk (1982) mentioned that, under optimal conditions, $P$. blanchardii had three full generations and a partial fourth one may be produced annually in humid and protected micro-environments. In Egypt, Hussain (1996) showed that, the population density of P. blanchardii on date palms at Bahria Oases has three distinct peaks in October, March and July.

\section{1- Distribution of insect population on the date palm leaflets}

The distribution of $F$. phoenicis on the different stratum of the date palm leaflets (Table, 3 \& Fig., 3) was varied in both studied years from one stratum to another. The basal stratum of the date palm leaflets received the highest infestation (37.4 - 38.3\%) and the apical stratum received the least infestation (26.4 - 28.2\%) whereas the middle stratum received intermediate infestation $(34.4-35.3 \%)$ for the $1^{\text {st }}$ and $2^{\text {nd }}$ 
year, respectively. The aforementioned results emphasize that, F. phoenicis prefers the basal stratum of date palm leaflets than middle or apical ones. In Iraq, Al-Hafidh et al. (1981) came to similar results.

Table 3. Distribution of $F$. phoenicis population on the different stratum of the date palm leaflets at Giza Governorate in the two years (2008/2009 \& 2009/2010).

\begin{tabular}{|c|c|c|c|c|}
\hline \multirow{2}{*}{ Stratum } & \multicolumn{4}{|c|}{ Mean number of insect population } \\
\cline { 2 - 5 } & $1^{\text {st }}$ year & $\%$ & $2^{\text {nd }}$ year & $\%$ \\
\hline Apical stratum & $217.29(\mathrm{c})$ & $28.23 \%$ & $166.54(\mathrm{c})$ & $26.43 \%$ \\
\hline Middle stratum & $265.00(\mathrm{~b})$ & $34.43 \%$ & $222.79(\mathrm{~b})$ & $35.34 \%$ \\
\hline Lower stratum & $287.50(\mathrm{a})$ & $37.35 \%$ & $240.88(\mathrm{a})$ & $38.26 \%$ \\
\hline Total population & 769.79 & - & 630.21 & - \\
\hline F value & \multicolumn{2}{|c|}{$22.7 * *$} & & $37.4 * *$ \\
\hline LSD at 5\% & \multicolumn{2}{|c|}{21.43} & \multicolumn{2}{c|}{18.04} \\
\hline
\end{tabular}

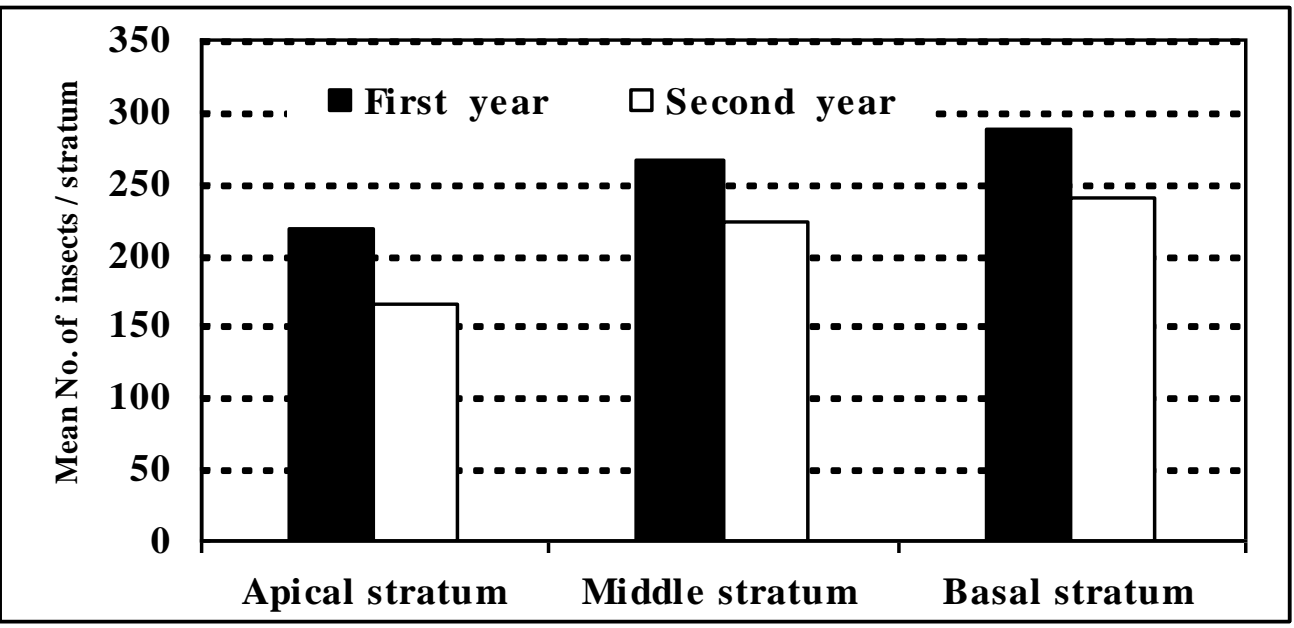

Fig. 5. Distribution of $F$. phoenicis population on the different stratum of the date palm leaflets at Giza Governorate in the two years (2008/2009 \& 2009/2010).

\section{V- Effect of main weather factors on the annual generations}

\section{I- The $1^{\text {st }}$ generation (Early summer generation)}

\section{A: Nymphal population}

\section{1-Effect of daily mean temperature}

Data in Tables (3\&4) showed a positive relation, highly significant ( $r=0.807$ \& 0.793 ) for the daily mean temperature on the nymphal activity during the $1^{\text {st }}$ generation in the both studied years. The partial regression coefficient showed insignificant effect for this factor on the nymphal activity in the $1^{\text {st }}$ generation in the 
two years ( $\mathrm{t}$ value $=1.10 \& 1.19$ ) when the daily mean relative humidity become around its mean. The obtained results revealed that, daily mean temperature within the optimum range of the nymphal activity in the $1^{\text {st }}$ generation in the both years, respectively.

\section{2- Effect of daily mean relative humidity (\%)}

Tables (3\&4) showed negative relation for daily mean relative humidity on the nymphal population in the both years, significant $(r=-0.770)$ in the $1^{\text {st }}$ year and highly significant $(r=-0.792)$ in the $2^{\text {nd }}$ one. The partial regression coefficient showed insignificant effect for this factor on the nymphal activity in the $1^{\text {st }}$ generation in the both years ( $t$ value $=-0.43 \&-1.18$ ) when the daily mean temperature become around its mean. The obtained results revealed that, daily mean relative humidity around the optimum range of nymphal activity in the $1^{\text {st }}$ generation in the both years, respectively.

Table 4. Effect of daily mean temperature and \%R.H. on F. phoenicis generations on date palm at Giza Governorate in the $1^{\text {st }}$ year (2008/2009).

\begin{tabular}{|c|c|c|c|c|c|c|c|c|c|c|c|c|}
\hline \multirow{2}{*}{ 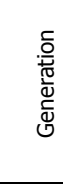 } & & \multirow{2}{*}{$\begin{array}{l}\text { Insect } \\
\text { stage }\end{array}$} & \multicolumn{2}{|c|}{$\begin{array}{c}\text { Generation } \\
\text { period }\end{array}$} & \multirow{2}{*}{ 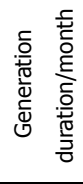 } & \multicolumn{2}{|c|}{ Weather factors } & \multirow{2}{*}{$\begin{array}{c}\text { Simple } \\
\text { Correlation } \\
r \\
\text { Value }\end{array}$} & \multicolumn{2}{|c|}{$\begin{array}{c}\text { Multi-regression } \\
\text { values }\end{array}$} & \multicolumn{2}{|c|}{$\begin{array}{l}\text { ANOVA } \\
\text { Table }\end{array}$} \\
\hline & & & From & To & & Factor & Mean & & $\begin{array}{l}\text { P. reg. * } \\
\pm \text { s.e }\end{array}$ & $\begin{array}{c}\mathrm{t} \\
\text { value }\end{array}$ & $\begin{array}{c}\mathrm{F} \\
\text { value }\end{array}$ & $\begin{array}{l}\text { E.V. } \\
\%\end{array}$ \\
\hline \multirow{4}{*}{$\stackrel{t_{n}}{\sim}$} & \multirow{4}{*}{ 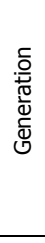 } & \multirow{2}{*}{ Nymph } & Early & Early & \multirow{2}{*}{4} & Mean temp. ${ }^{\circ} \mathrm{C}$ & $21.2^{\circ} \mathrm{C}$ & $0.807^{* *}$ & $24.4 \pm 7.2$ & 1.10 & \multirow{2}{*}{$5.9^{*}$} & \multirow{2}{*}{66.1} \\
\hline & & & Mar. & Jul. & & Mean \%R.H. & $61.7 \%$ & $-0.770^{*}$ & $-25.3 \pm 8.7$ & -0.43 & & \\
\hline & & \multirow{2}{*}{ Adult } & Early & Early & \multirow{2}{*}{4} & Mean temp. ${ }^{\circ} \mathrm{C}$ & $21.2^{\circ} \mathrm{C}$ & $0.692^{*}$ & $26.6 \pm 9.1$ & 1.40 & \multirow{2}{*}{2.9} & \multirow{2}{*}{49.2} \\
\hline & & & Mar. & Jul. & & Mean \%R.H. & $61.7 \%$ & -0.573 & $19.7 \pm 5.1$ & 0.39 & & \\
\hline \multirow{4}{*}{$\stackrel{g}{N}$} & \multirow{4}{*}{ 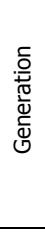 } & \multirow{2}{*}{ Nymph } & Early & Early & \multirow{2}{*}{3} & Mean temp. ${ }^{\circ} \mathrm{C}$ & $28.6^{\circ} \mathrm{C}$ & -0.484 & $-38.7 \pm 6.8$ & -0.63 & \multirow{2}{*}{3.8} & \multirow{2}{*}{65.4} \\
\hline & & & Jul. & Oct. & & Mean \%R.H. & $63.5 \%$ & $0.787^{*}$ & $65.2 \pm 19.6$ & 2.20 & & \\
\hline & & \multirow{2}{*}{ Adult } & Early & Early & \multirow{2}{*}{3} & Mean temp. ${ }^{\circ} \mathrm{C}$ & $28.6^{\circ} \mathrm{C}$ & -0.499 & $-35.7 \pm 5.9$ & -0.69 & \multirow{2}{*}{3.5} & \multirow{2}{*}{63.8} \\
\hline & & & Jul. & Oct. & & Mean \%R.H. & $63.5 \%$ & $0.771^{*}$ & $51.6 \pm 10.9$ & 2.07 & & \\
\hline \multirow{4}{*}{$\stackrel{D}{m}$} & \multirow{4}{*}{ 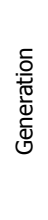 } & & Mid- & Early & \multirow{2}{*}{4.5} & Mean temp. ${ }^{\circ} \mathrm{C}$ & $21.6^{\circ} \mathrm{C}$ & $0.876^{* *}$ & $45.5 \pm 8.6$ & $2.7^{*}$ & \multirow{2}{*}{$12.0^{* *}$} & \multirow{2}{*}{77.4} \\
\hline & & торит & Sep. & Feb. & & Mean \%R.H. & $65.9 \%$ & $-0.732^{*}$ & $-26.9 \pm 5.7$ & -0.48 & & \\
\hline & & \multirow[b]{2}{*}{ Adult } & Mid- & Early & \multirow[b]{2}{*}{4.5} & Mean temp. ${ }^{\circ} \mathrm{C}$ & $21.6^{\circ} \mathrm{C}$ & $0.796^{* *}$ & $31.9 \pm 5.5$ & 2.10 & \multirow{2}{*}{$6.1^{*}$} & 5 \\
\hline & & & & Feb. & & Mean \%R.H. & $65.9 \%$ & -0.643 & $-9.7 \pm 2.5$ & -0.19 & & | \\
\hline
\end{tabular}

*P. reg. = Partial regression coefficient. 
Table 5. Effect of daily mean temperature and \%R.H. on F. phoenicis generations on date palm at Giza Governorate in the 2nd year (2009/2010).

\begin{tabular}{|c|c|c|c|c|c|c|c|c|c|c|c|}
\hline \multirow{2}{*}{ 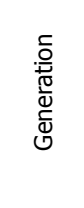 } & \multirow{2}{*}{$\begin{array}{l}\text { Insect } \\
\text { stage }\end{array}$} & \multicolumn{2}{|c|}{$\begin{array}{c}\text { Generation } \\
\text { period }\end{array}$} & \multirow{2}{*}{ 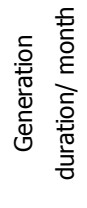 } & \multicolumn{2}{|c|}{ Weather factors } & \multirow{2}{*}{$\begin{array}{c}\begin{array}{c}\text { Simple } \\
\text { correlation }\end{array} \\
r \\
\text { value }\end{array}$} & \multicolumn{2}{|c|}{$\begin{array}{c}\text { Multi-regression } \\
\text { values }\end{array}$} & \multicolumn{2}{|c|}{$\begin{array}{l}\text { ANOVA } \\
\text { Table }\end{array}$} \\
\hline & & From & To & & Factor & Mean & & $\begin{array}{l}\text { P. reg. * } \\
\pm \text { s.e }\end{array}$ & $\begin{array}{c}\mathrm{t} \\
\text { value }\end{array}$ & $\begin{array}{c}\mathrm{F} \\
\text { value }\end{array}$ & $\begin{array}{c}\text { E.V. } \\
\%\end{array}$ \\
\hline \multirow{4}{*}{ 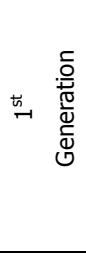 } & \multirow[t]{2}{*}{ Nymph } & \multirow{2}{*}{$\begin{array}{l}\text { Early } \\
\text { Mar. }\end{array}$} & \multirow{2}{*}{$\begin{array}{l}\text { Mid- } \\
\text { Jul. }\end{array}$} & \multirow[t]{2}{*}{4.5} & $\begin{array}{l}\text { Mean temp. } \\
{ }^{\circ} \mathrm{C}\end{array}$ & $20.9^{\circ} \mathrm{C}$ & $0.793^{* *}$ & $12.6 \pm 4.6$ & 1.19 & \multirow[t]{2}{*}{$7.8^{*}$} & \multirow[t]{2}{*}{69} \\
\hline & & & & & Mean \%R.H. & $63.0 \%$ & $-0.792^{* *}$ & $-28.4 \pm 6.1$ & -1.18 & & \\
\hline & \multirow[t]{2}{*}{ Adult } & \multirow{2}{*}{$\begin{array}{l}\text { Early } \\
\text { Mar. }\end{array}$} & \multirow{2}{*}{$\begin{array}{l}\text { Mid- } \\
\text { Jul. }\end{array}$} & \multirow[t]{2}{*}{4.5} & $\begin{array}{l}\text { Mean temp. } \\
{ }^{\circ} \mathrm{C}\end{array}$ & $20.9^{\circ} \mathrm{C}$ & $0.679^{*}$ & $94.7 \pm 19.5$ & 1.00 & \multirow[t]{2}{*}{3.2} & \multirow[t]{2}{*}{48.1} \\
\hline & & & & & Mean \%R.H. & $63.0 \%$ & $-0.638^{*}$ & $-12.6 \pm 3.6$ & -0.52 & & \\
\hline \multirow{4}{*}{ 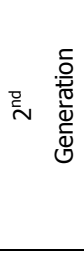 } & \multirow[t]{2}{*}{ Nymph } & \multirow{2}{*}{$\begin{array}{l}\text { Early } \\
\text { Jul. }\end{array}$} & \multirow{2}{*}{$\begin{array}{l}\text { Mid- } \\
\text { Sep. }\end{array}$} & \multirow[t]{2}{*}{2.5} & $\begin{array}{l}\text { Mean temp. } \\
{ }^{\circ} \mathrm{C}\end{array}$ & $27.9^{\circ} \mathrm{C}$ & 0.720 & $36.5 \pm 8.6$ & 0.45 & \multirow[t]{2}{*}{4.3} & \multirow[t]{2}{*}{74} \\
\hline & & & & & Mean \%R.H. & $64.8 \%$ & $0.850^{*}$ & $39.0 \pm 8.3$ & 1.60 & & \\
\hline & \multirow[t]{2}{*}{ Adult } & \multirow{2}{*}{$\begin{array}{l}\text { Early } \\
\text { Jul. }\end{array}$} & \multirow{2}{*}{$\begin{array}{l}\text { Mid- } \\
\text { Sep. }\end{array}$} & \multirow[t]{2}{*}{2.5} & $\begin{array}{l}\text { Mean temp. } \\
{ }^{\circ} \mathrm{C}\end{array}$ & $27.9^{\circ} \mathrm{C}$ & 0.750 & $37.0 \pm 7.4$ & 0.58 & \multirow[t]{2}{*}{5.5} & \multirow[t]{2}{*}{78.4} \\
\hline & & & & & Mean \%R.H. & $64.8 \%$ & $0.872^{*}$ & $34.1 \pm 9.4$ & 1.75 & & \\
\hline \multirow{4}{*}{ 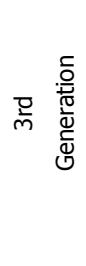 } & \multirow[t]{2}{*}{ Nymph } & Early & Mid- & 4.5 & $\begin{array}{l}\text { Mean temp. } \\
{ }^{\circ} \mathrm{C}\end{array}$ & $22.0^{\circ} \mathrm{C}$ & $0.769^{*}$ & $23.9 \pm 5.5$ & 1.45 & $5.5^{*}$ & 60.9 \\
\hline & & & Jain. & & Mean \%R.H. & $66.3 \%$ & $-0.702^{*}$ & $-23.9 \pm 4.0$ & -0.56 & & \\
\hline & Adult & Early & Mid- & 4.5 & $\begin{array}{l}\text { Mean temp. } \\
{ }^{\circ} \mathrm{C}\end{array}$ & $22.0^{\circ} \mathrm{C}$ & 0.562 & $14.6 \pm 3.5$ & 0.97 & 36 & 86 \\
\hline & & Sep. & Jan. & & Mean \%R.H. & $66.3 \%$ & -0.473 & $-3.7 \pm 1.6$ & -0.10 & & \\
\hline
\end{tabular}

* P. reg. = Partial regression coefficient

\section{3- The combined effect of the daily mean temperature and relative}

\section{humidity}

The combined effect (Tables, 3\&4) of both daily mean temperature and relative humidity showed significant effect $(F=5.9 \& 7.8)$ on the nymphal population in the $1^{\text {st }}$ generation in the two years, respectively. The changes in the half monthly counts of the nymphal population referred to the effect of the tested weather factors ranged $66.1-69 \%$ in both years, respectively.

\section{B: Adult population}

\section{1-Effect of daily mean temperature}

Daily mean temperature (Tables, 3\&4) showed positive relation, significant on the adult activity in the $1^{\text {st }}$ generation in the both years $(r=0.692 \& 0.679)$, respectively. Partial regression coefficient showed insignificant effect for this factor on the adult activity in the both years $(t=1.40 \& 1.00)$ when the daily mean relative humidity become around its mean. The obtained results revealed that, daily mean temperature within the optimum range of adult activity in both years, respectively. 


\section{2- Effect of daily mean relative humidity}

Daily mean relative humidity (Tables, $3 \& 4$ ) had negative relation, insignificant ( $r$ $=-0.573)$ on the adult population in the $1^{\text {st }}$ year and significant $(r=-0.638)$ in the $2^{\text {nd }}$ one. Partial regression coefficient showed insignificant effect for this factor on the adult activity in the both years ( $\mathrm{t}$ value $=0.39 \&-0.52$ ) when daily mean temperature become around its mean. The obtained results revealed that, daily mean relative humidity under the optimum range of adult activity in the $1^{\text {st }}$ year and around the optimum range in the $2^{\text {nd }}$ one.

\section{3- The combined effect of the daily mean temperature and relative humidity}

The combined effect (Tables, 3\&4) of both daily mean temperature and relative humidity on the adult activity in the $1^{\text {st }}$ generation was insignificant in the both years $(F=2.9$ \&3.2). The changes in the half monthly counts of the adult population referred to the effect of the two tested weather factors ranged $48.1-49.2 \%$ in the two years.

\section{II- The $2^{\text {nd }}$ generation (Late summer generation)}

\section{A: Nymphal population}

\section{1- Effect of daily mean temperature}

Daily mean temperature (Tables, 3\&4) showed insignificant relation on the nymphal activity in the $2^{\text {nd }}$ generation, negative $(r=-0.484)$ in the $1^{\text {st }}$ year and positive $(r=0.720)$ in the second one. Partial regression coefficient showed insignificant effect for this factor on the nymphal activity in the $2^{\text {nd }}$ generation in the both years ( $t$ value $=-0.63 \& 0.45$ ) when the daily mean relative humidity become around its mean. The obtained results revealed that, daily mean temperature become around the optimum range of nymphal activity in the $1^{\text {st }}$ year and under the optimum range in the $2^{\text {nd }}$ year, respectively.

\section{2- Effect of daily mean relative humidity}

Daily mean relative humidity (Tables, 3\&4) showed significant positive relation ( $r$ $=0.787 \& 0.850$ ) on the nymphal population in the $2^{\text {nd }}$ generation in the two studied years. Partial regression coefficient showed insignificant effect for this factor on the nymphal activity in the $2^{\text {nd }}$ generation in the both years ( $t$ value $=2.20 \& 1.60$ ) when the daily mean temperature become around its mean. The obtained results revealed that, daily mean relative humidity within the optimum range of the nymphal activity in the two years, respectively.

\section{3- The combined effect of the daily mean temperature and relative humidity}

The combined effect (Tables, 3\&4) of both daily mean temperature and relative humidity showed insignificant effect $(F=3.8 \& 4.3)$, on the nymphal population the $2^{\text {nd }}$ 
generation in the both years respectively. The changes in the half monthly counts of the nymphal population referred to the effect of the tested weather factors ranged $65.4-74.0 \%$ in both years, respectively.

\section{B: Adult population}

\section{1- Effect of daily mean temperature}

Daily mean temperature (Tables, 3\&4) showed negative relation on the adult activity, insignificant in the $1^{\text {st }}$ year $(r=-0.484)$ and positive relation insignificant in the $2^{\text {nd }}$ one $(r=0.720)$. Partial regression coefficient showed insignificant effect for this factor on the adult activity in the $2^{\text {nd }}$ generation in the both years ( $t$ value $=-0.69 \&$ 0.58 ) when the daily mean relative humidity become around its mean. The obtained results revealed that, daily mean temperature was around the optimum range of the adult activity in the $1^{\text {st }}$ year and under the optimum range in the $2^{\text {nd }}$ one, respectively.

\section{2- Effect of daily mean relative humidity}

Data in Tables (3\&4) showed that, daily mean relative humidity had positive relation and significant on the adult population in the both years $(r=0.771 \& 0.872$ ). Partial regression coefficient showed insignificant effect for this factor on the adult activity in the $2^{\text {nd }}$ generation in the both years ( $t$ value $=2.07 \& 1.75$ ) when the daily mean temperature become around its mean. The obtained results revealed that, daily mean relative humidity proved to be within the optimum range of adult activity in the both studied years, respectively.

\section{3- The combined effect of the daily mean temperature and relative humidity}

The combined effect (Tables, 3\&4) of both daily mean temperature and relative humidity showed insignificant effect $(F=3.5 \& 5.5)$ on the adult activity in the $2^{\text {nd }}$ generation in the two years, respectively. The changes in the half monthly counts of the adult population referred to the effect of the two tested weather factors ranged $63.8-78.4 \%$ for both years, respectively.

\section{III- The $3^{\text {rd }}$ generation (autumn generation)}

\section{A: Nymphal population}

\section{1- Effect of daily mean temperature}

Daily mean temperature (Tables, $3 \& 4$ ) showed positive relation on the nymphal activity in the $3^{\text {rd }}$ generation, highly significant in the $1^{\text {st }}$ year $(r=0.876)$ and significant $(r=0.769)$ in the $2^{\text {nd }}$ one. Partial regression coefficient showed significant effect for this factor on the nymphal activity in the $3^{\text {rd }}$ generation ( $t$ value $=2.7$ ) in the $1^{\text {st }}$ year and insignificant effect ( $t$ value $=1.45$ ) in the $2^{\text {nd }}$ one when the daily mean relative humidity become around its mean. The obtained results revealed that, daily 
mean temperature under the optimum range of nymphal activity in the $1^{\text {st }}$ year and within the optimum range in the $2^{\text {nd }}$ year.

\section{2- Effect of daily mean relative humidity}

Daily mean relative humidity (Tables, $3 \& 4$ ) showed negative relation, significant effect $(r=-0.732 \&-0.702)$ on the nymphal population in the $3^{\text {rd }}$ generation in the two years, respectively. However, partial regression coefficient showed insignificant effect for this factor on the nymphal activity in the $3^{\text {rd }}$ generation in the both years ( $t$ value $=-0.48 \&-0.56$ ) when the daily mean temperature become around its mean. The obtained results revealed that, daily mean relative humidity around the optimum range of the nymphal activity in the two years, respectively.

\section{3- The combined effect of the daily mean temperature and relative humidity}

The combined effect (Tables, 3\&4) of both daily mean temperature and relative humidity on the nymphal population in the $3^{\text {rd }}$ generation was highly significant ( $\mathrm{F}=$ $12.0)$ in the $1^{\text {st }}$ year and significant $(F=5.5)$ in the $2^{\text {nd }}$ one, respectively. The changes in the half monthly counts of the nymphal population referred to the effect of the tested weather factors ranged $60.9-77.4 \%$ in the both years, respectively.

\section{B: Adult population}

\section{1- Effect of daily mean temperature}

Daily mean temperature (Tables, $3 \& 4$ ) showed positive relation on the adult activity in the $3^{\text {rd }}$ generation, highly significant $(r=0.796)$ in the $1^{\text {st }}$ year and significant $(r=0.562)$ in the $2^{\text {nd }}$ one. However, partial regression coefficient showed insignificant effect for this factor on the adult activity in the $3^{\text {rd }}$ generation in the both years $(t$ value $=2.10 \& 0.97$ ) when the daily mean relative humidity become around its mean. The obtained results revealed that, daily mean temperature within the optimum range of the adult activity in the both years, respectively.

\section{2- Effect of daily mean relative humidity}

Data in Tables (3\&4) showed that, daily mean relative humidity had negative relation and insignificant on the adult population in the both years $(r=-0.643 \&$ 0.473), respectively. Partial regression coefficient showed insignificant effect for this factor on the adult activity in the $3^{\text {rd }}$ generation in the both years ( $t$ value $=-0.19 \&-$ 0.10) when the daily mean temperature become around its mean. The obtained results revealed that, daily mean relative humidity around the optimum range of adult activity in the both years, respectively. 


\section{3-The combined effect of the daily mean temperature and relative humidity}

The combined effect (Tables, 3\&4) of both daily mean temperature and relative humidity on the adult activity in the $3^{\text {rd }}$ generation was significant $(F=6.1)$ in the $1^{\text {st }}$ year and insignificant effect $(F=3.6)$ in the $2^{\text {nd }}$ one, respectively. The changes in the half monthly counts of the adult population referred to the effect of the two tested weather factors ranged $48.6-63.5 \%$ for both years, respectively.

Laudeho \& Benassy (1969) stated that the density and severity of infestation with $P$. blanchardii is affected by microclimate conditions. High temperature combined with wind and low humidity was very effective for the survival of the crawlers.

\section{REFERENCES}

1. Abdel-Rahman, M. and M. A. Amro. 2007. Analytical review of the main arthropod pests and natural enemies associated with date palm trees in Egypt. Egypt. J. Agric. Res., 85 (1): 99-117.

2. Al-Hafidh, E. M. T., I . A. Swair and A. Abdallah. 1981. Ecological studies and chemical control of parlatoria date scale insect, Parlatoria blanchardii (Targ.) (Homoptera - Diaspididae) on date palms in Iraq. Date palm J., 1(1): 117-128.

3. Balachowsky, A. S. 1967. Une espèce nouvelle de Fiorinia (Coccoidea - Diaspidini) vivant sur palmeir-dattier dans les Oasis du sud de I’Iran.Ann. Soc. Ent. France (n. s.), 3:771-775.

4. El-Haidari, H.S. 1980. Insect of dates and date palms in the Near East and North Africa. Regional Project for palm and dates Research Center in the Near East and North Africa. Baghdad, Iraq. pp.37.

5. El-Haidari, H.S. and E. M. T. Al-Hafidh.1986. Palm and date arthropod pests in the Near East and North Africa. Regional Project for palm and dates Research Center in the Near East and North Africa. Baghdad, Iraq. pp.126.

6. Elwan, E. A. 2000. Survey of the insect and mite pests associated with date palm trees in Al-Dakhliya Region, Sultanate of Oman. Egypt. J. Agric. Res., 78(2):653664.

7. Ghabbour, M .W. and Z. K. Mohammad . 2010. Fiorinia phoenicis (Hemiptera: Coccoidea: Diaspididae) new pest of palm trees in Egypt. J. Egypt. Ger. Soc. Zool., 58(E): 15-20.

8. Ghabbour, M .W., Z. K. Mohammad and E. A. Elwan . 1996. Fiorinia linderae Takagi new record in Oman (Homoptera : Diaspididae). J. Egypt. Ger. Soc. Zool., 19(E): 51-58. 
9. Hussain, A. A. 1974 . Date palms and dates with their pests in Iraq. Univ. Baghdad, Ministry of Higher Educ. Sci. Res. Baghdad, 244 pp.

10. Hussain, A. E. 1996. Comparative study on distribution and seasonal abundance of date scale, Parlatoria blanchardii (Targ.) populations in Giza and Bahria Oases regions. Bull. Ent. Soc. Egypt., 74:54-60.

11. Laudeho, Y. and C. Benassy. 1969. Contribution a letude del ecologie de Parlatoria blanchardii (Targ.) on Adrar Mauritanian. Fruits, 24:273-287.

12. Martin, H. 1958. Pests and diseases of date palm in Libya. FAO Plant Prot. Bull., 6(8):120-123.

13. Matile, F. D. 1984c. Insects of Saudi Arabia Homoptera: Suborder Coccoidea. Fauna of Saudi Arabia, 6:219-228.

14. Sharif, M. and I. Wajih. 1982. Date palm pests and diseases in Pakistan. Proceedings of the first symposium on the date palm, Held at King Faisal University, Al-Hassa, Saudi Arabia, March 23-25, P: 440-451.

15. Smirnoff, W. A. 1957. La cochenille du palmier dattier Parlatoria blanchardii (Targ.) en Afrique du Nord. Comportment, importance economique, predateurs et lutte biologique. Entomophaga, 2:1-99.

16. Takagi, S. and M. Moghaddam . 2005. New or noteworthy armoured scale insects occurring in Iran (Homoptera: Coccoidea: Diaspididae). Ins. Matsum. n. s. 61:4374.

17. Talhouk, A. S. 1982. The present status of date palm pests in Saudi Arabia. Proceedings of the first symposium on the date palm, Held at King Faisal University, Al-Hassa, Saudi Arabia, March 23-25, P:432-438. 
Fiorinia phoenicis النشاط الموسمي لحشرة النخيل القشرية البنية المبططة على نخيل البلح في محافظة الجيزة

\author{
السيد عبد الحميد علوان مها إبراهيم السيد عادل محمد سراج \\ معرج بحوث وقابة النباتات - مركز البحوث الزراعية - الدقى - جيزة \\ Email: Dr.Sayedelwan@Yahoo.com
}

تعتبر الحشرات القترية من الافات الحشرية الهامة التى تصيب نخيل البلح فى مصر • سجلت حشرة النخيل القشرية البنية المبططة Fiorinia phoenicis Balachowsky فى السنوات الاخيرة بأعداد كثيرة على سعف وثمار نخيل البلح فى بعض محافظات مصر ـ تسبب الاصنابة الثديدة بالحشرة اصفر ار وجفاف السعف وتثوة الثمار ـ أجريت الدر ساسة الحالية على نخيل البلح بمركز البحوث الزر اعية - محافظة الجيزة لمدة عامين متتاليين ( مارس 2008 - فبر اير 2010) بغرض دراس استة النشاط

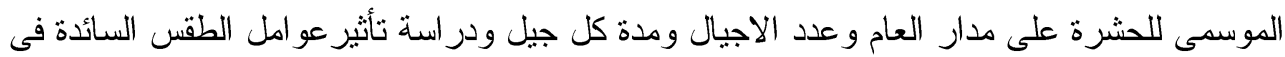

منطقة الدر اسة على نشاط الحشرة بغرض وضع برنامج متكامل لمكافحتها و الحد من انتشار ها. اتضح من نتائج الدراسة وجود ثلاثة اجيال متداخلة للحشرة على مدار العام ، ظهر الجيل الاول

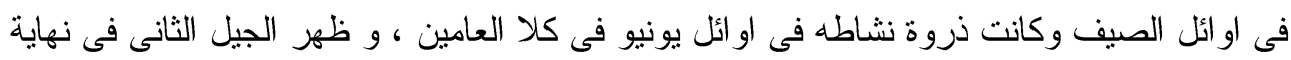
الصيف وكانت ذروة نشاطه فى اغسطس/سبتمبر من العامين ، وظهر الجيل الثالث فى الخريف وكانت

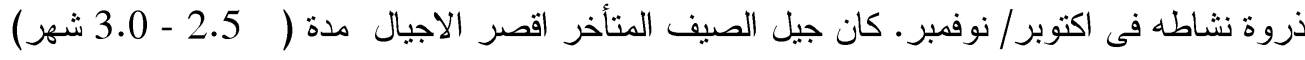

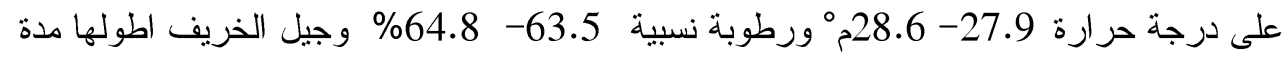

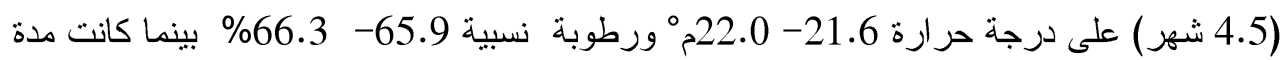

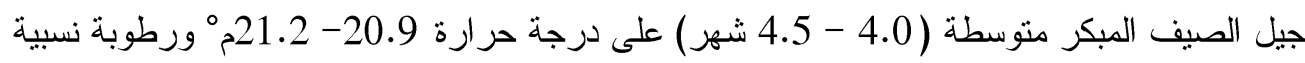
62.5- 63.0\% كان جيل الخريف اقوى الاجيال (944.7 - 1016.0 حشرة/خوصة بمتوسط قدرة

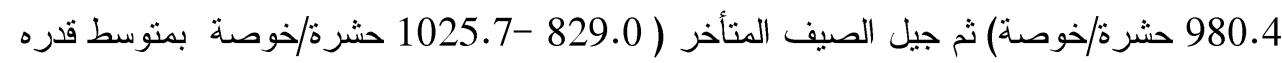

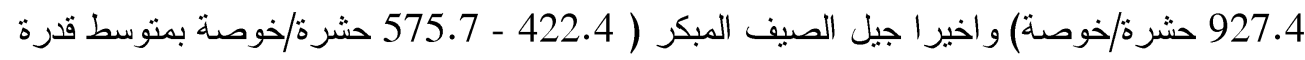
499.1 حشرة/خوصة).

وتبين من الدراسة وجود الحشرة على سعف النخيل على مدار العام و انخفض تعدادها بدرجة

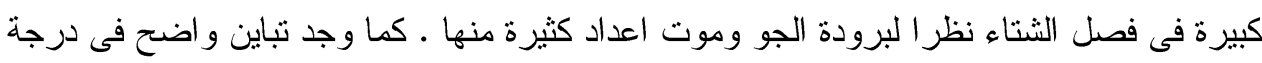
انتشار الحشرة على الخوص حيث وجدت اعداد كثيرة من اطوار الحشرة على الثلث السفلى من

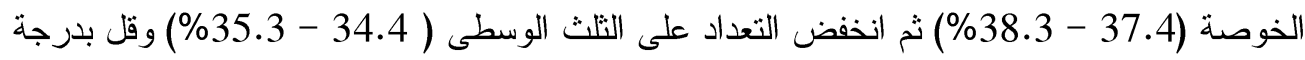
كبيرة على التلث الطرفى من الخوصة (26.4 - 28.2\%) فى كلا العامين على التو الى. كما تأثز نشاط

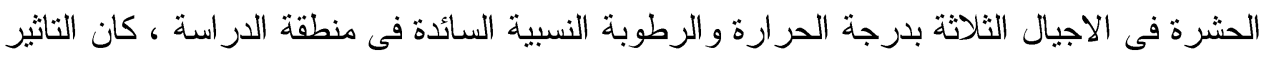
المشترك للعاملين (متوسط درجة الحر ارة و الرطوبة النسبية) متباينا على نشاط الحورية و الحشرة الكاملة

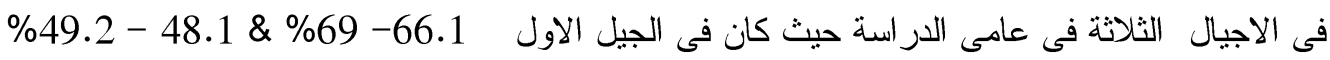
وفى الجيل الثانى 65.4 - 74.0\% \& 63.8 - 78.4\% وفى الجيال

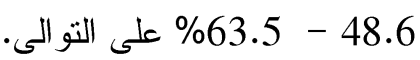

